\title{
Cost-effectiveness analysis of subcutaneous immunoglobulin replacement therapy in Iranian patients with primary immunodeficiencies
}

\author{
Hosein Shabaninejad ${ }^{1}$, Asra Asgharzadeh ${ }^{2 *}$, Aziz Rezapour ${ }^{3}$, Nima Rezaei ${ }^{4}$ \\ Received: 26 Jul 2016 \\ Published: 17 Dec 2017
}

\section{Abstract}

Background: Economic evaluation of subcutaneous immunoglobulin therapy (SCIG) is important, and it has recently been used for treatment of patients with primary immunodeficiency (PID) diseases, and can improve allocation of resources in health care systems. The present research aimed at providing an economic assessment of SCIG and IVIG (intravenous immunoglobulin therapy) administration in Iran.

Methods: Data related to clinical effectiveness were obtained from a meta-analysis. Economic analysis was performed taking into account the perspective of health care providers. Incremental cost-effectiveness ratio (ICER) was applied for economic evaluation of the 2 methods, and GDP per capita was considered as a threshold.

Results: The results of meta-analysis suggested a higher effectiveness of SCIG compared to IVIG in serum immunoglobulin (Ig) levels $(\mathrm{SMD}=0.336)$ and adverse effects $(\mathrm{OR}=0.497)$, while the cost of IVIG was higher than SCIG $(\$ 1370$ vs. $\$ 121)$. The ICER obtained in this paper (\$2939 for adverse effects and \$4348 for serum Ig level) was less than the GDP per capita in Iran $(\$ 4,916.10)$, and thus SCIG is a more cost-effective therapy for PID patients.

Conclusion: Switching from IVIG to SCIG is cost-effective for PID patients and is approved by the health care providers' points of view in this study.

Keywords: Subcutaneous immunoglobulin, Intravenous immunoglobulin, Primary immunodeficiency, Cost-effectiveness

Copyright $\odot$ Iran University of Medical Sciences

Cite this article as: Shabaninejad H, Asgharzadeh A, Rezapour A, Rezaei N. Cost-effectiveness analysis of subcutaneous immunoglobulin replacement therapy in Iranian patients with primary immunodeficiencies. Med J Islam Repub Iran. 2017 (17 Dec);31:94. https://doi.org/10.14196/mjiri.31.94

\section{Introduction}

Primary immunodeficiency diseases (PIDs) are caused by defects of the immune system. As a result, the body cannot protect itself against bacterial, viral, or fungal infections $(1,2)$. Based on statistics, 25 in 1 million people have PID (3). In Iran, there are 1661 recorded cases of PID in children medical centers. Nevertheless, $70 \%$ to $90 \%$ of these PID patients are unidentified, as they may die before the disease is diagnosed (4).

Immunoglobulin replacement is an important therapy for a variety of conditions in the fields of dermatology,

Corresponding author: Asra Asgharzadeh, as.asgharzadeh@gmail.com

1. Department of Health Management, School of Health Management \& Information Sciences, Iran University of Medical Sciences, Tehran, Iran.

2. School of Health Management \& Information Sciences, Iran University of Medical Sciences, Tehran, Iran, \& Health Technology Assessment Group (HTAG), Universal Scientific Education and Research Network (USERN), Tehran, Iran.

3. Health Management and Economics Research Center, Iran University of Medical Sciences, Tehran, Iran.

4. Research Center for Immunodeficiencies Children's Medical Center, Tehran University of Medical Sciences, Tehran, Iran, \& Department of Immunology and Biology, School of Medicine Tehran University of Medical Sciences, Tehran, Iran, \& Network of Immunity in Infection, Malignancy and Autoimmunity (NIIMA) Universal Scientific Education and Research Network (USERN), Tehran, Iran. neurology, hematology, and immunology (5). For most of PID patients, it is the only life-saving therapy, and treatment is life-long. Since the vast majority of these patients have primary antibody failure, successful treatment depends on multiple factors, such as availability of products, type of immunodeficiency, and any comorbidities of the patient. Essential components include long-term followup, regular monitoring, and a close relationship between the patient and the multidisciplinary clinical immunology team (6).

$\uparrow$ What is "already known" in this topic:

Economic evaluation of subcutaneous immunoglobulin therapy (SCIG) and IVIG (intravenous immunoglobulin therapy) were conducted by researchers to treat patients with primary immunodeficiency (PID) in Iran; and the results revealed that SCIG is a more cost-effective therapy for PID patients.

\section{$\rightarrow$ What this article adds:}

The present research provided a cost- effectiveness analysis of SCIG and IVIG administration in Iran; and the results revealed that switching from IVIG to SCIG is cost-effective for PID patients and is approved by the health care providers' points of view in this study. 
IVIG products are currently available in different concentrations and compositions and can deliver up to $2 \mathrm{~g} / \mathrm{kg}$ or more per infusion, with few adverse effects. IVIG preparations have improved over the years and have evolved from immune serum globulin, which is injected intramuscularly or subcutaneously at relatively low doses (100-150 $\mathrm{mg} / \mathrm{kg}$ per month) (7). Most PID patients do not experience serious adverse effects from immunoglobulins. Some patients experience such symptoms as headache, dizziness, fever, chills, nausea, vomiting, or pain in the muscles or the back. More serious adverse effects are very rare (8) and are less frequent in patients treated with SCIG than IVIG. Subcutaneous infusion can sometimes cause swelling and pain in the injection site (9).

Several studies in different countries compared these 2 methods with respect to costing and found different results, which may reflect the characteristics of different health care systems. However, most of studies concluded that SCIG delivery is less costly than IVIG administration. An economic evaluation study in Canada indicated less cost differences between these 2 methods (10), while another study from Canadian health care perspective revealed that using SCIG method decreases $78 \%$ of costs compared with IVIG method (11). Moreover, studies from Germany and the United States revealed that moving from IVIG to SCIG administration will decrease 50\% and $88 \%$ of costs, respectively $(12,13)$. In addition, studies in Sweden, Japan, and France showed less costs in SCIG than IVIG method (14-16). This study aimed at determining the cost-effectiveness of SCIG delivery at home compared with IVIG administration at hospital for PI patients from the perspective of the Iranian health care system.

\section{Methods}

This research provided a cost-effectiveness analysis of IVIG and SCIG, with an emphasis on changes in serum immunoglobulin (Ig) levels and adverse effects following therapy. The epidemiological model included PID patients, who received IVIG at Children's Medical Center of Tehran during 2014 and 2015, and costing was done from the perspective of the health care providers. Effectiveness of interventions was measured based on the results of a systematic review and meta-analysis of 24 primary studies (randomized control trials and cohort studies). IVIG costs were determined through field studies, and cost items were identified based on IVIG treatment protocol and hospital bills. While SCIG method has not yet been administered in Iran, we used previous studies to find the cost items of SCIG administration $(11,15)$. In this research, IVIG was compared to rapid push SCIG. Rapid push SCIG is a more common technique than pump infusion, and the patient can administer it directly with a syringe (17). To calculate the costs in one-year time horizon, we used average number of therapy sessions (12-16 sessions per year/4-6 hours per session for IVIG therapy and 48-64 sessions per year/1-2 hours per session for SCIG therapy). Finally, the total direct medical costs (cost of hospital, personnel, and medical supplies) were calculated for each method. Moreover, due to similar level of Ig doses in both therapies (the difference is in the number of injections), the costs of Ig products were not considered.

\section{Discounting}

In this study, costs were converted from Iranian Rial to US dollar's value in 2015 ( 1 Rial $=0.000033$ US Dollar), and due to the one-year time horizon, discount rate was not taken into account in calculations.

\section{Statistical analysis}

Meta-analysis with random effects and the MantelHaenszel method were performed using STATA 12.0 to combine the results of the systematic review (1991 to March 16, 2015) of the studies that examined adverse effects and serum Ig level in IVIG and SCIG. Odds ratio (OR) for dichotomous variable (adverse effects) was extracted from the studies and calculated with 95\% confidence interval. Random effect model was used for data related to serum Ig levels, and mean differences were used for measurement.

For economic modeling, the interventions were defined based on standards and a decision tree, which was developed using TreeAge 2011, was used to determine direct medical costs with a one-year time horizon (first year of treatment). Parameters with the greatest effect on costs, such as the number of injections and cost of hospital, personnel and medical supplies were examined using sensitivity analysis. Finally, incremental cost-effectiveness ratio (ICER) from each effectiveness index was used for cost-effectiveness analysis. Due to lack of a threshold in Iran, GDP per capita was used as the upper bound.

\section{Results}

Standard IVIG therapy usually involves 1 infusion per month and the maintenance dose for PID patients is usually 400 to $600 \mathrm{mg} / \mathrm{kg}$, which is administered every 3 or 4 weeks (18). In other words, patients are admitted to the hospital as outpatients every 21 to 28 days and receive the required dose within 4 to 6 hours (15). Subcutaneous infusion takes 1 to 2 hours, but the frequency of infusions is at least once a week. The patient or his/her parents can use this method of infusion; however, only trained individuals can administer this method (19).

\section{Effectiveness}

After searching related medical databases based on inclusion/exclusion criteria, 24 randomized control trials and cohort studies were systematically reviewed. A total of 945 patients were examined in this study (Serum Ig levels in 446 patients, infections and antibiotic use in 376 patients, and adverse effects in 431). The calculated OR indicated the advantage of IVIG over SCIG due to fewer adverse effects $(\mathrm{OR}=0.497 ; 95 \% \quad \mathrm{CI}: 0.180-171$; $\mathrm{p}<0.001)$. The results of the meta-analysis clearly revealed that SCIG (whether rapid push or pump) has more adverse effects than IVIG. Due to the heterogeneity of the studies (I-squared $=87.3 \%$ ), we used random model for metaanalysis. However, the infusion-site reactions in SCIG are moderate and tolerable. On the other hand, systemic adverse effects, such as headache, fever, and anaphylactic reactions, can occur in IVIG, which can be reduced by 
switching to SCIG. This can also improve tolerability in patients who have previously experienced systemic reactions to IVIG. Data related to switching to SCIG and changes in serum Ig levels were combined using metaanalysis, and the final results were expressed as standard mean difference (SMD). The obtained SMD was 0.336, indicating the higher effectiveness of SCIG in increasing serum Ig levels (20).

\section{Economic evaluation}

SCIG is an infusion technique that divides the monthly dose of IVIG into 4 weekly doses, while maintaining the same required Ig level. Patients are trained before beginning self-administration and are monitored weekly by local staff (21). Four to 6 training sessions are required for home-based administration of SCIG by the patient or their families, and they should be supported and offered regular medical and nursing follow- up care for continuous monitoring, advice, and clinical assessment (10). PID patients, who switch from IVIG to SCIG, need medical care only during the first 6 months, and a nurse is required to train the patients on SCIG method (6 hours during the first year, and yearly follow-up of 6 hours per year). SCIG treatment involves 3 training sessions and 4 weekly infusions using an infusion set (48 infusions per year) .IVIG treatment requires a nurse, a unit clerk, a scheduling clerk, and a ward aid (22). Patients' general characteristics are displayed in Table 1.

Due to lack of data on SCIG in Iran, a decision tree was developed for this intervention using secondary data (Fig. $1)$.

The assumptions are summarized as follow:

- Scheduling meetings varies based on the desired protocol, but generally 1 session every 3 or 4 weeks for life (IVIG) is enough.

- Cost of equipment and supplies in the treatment of venous include inpatient beds in hospital.

- Mean number of visits are 15 sessions per year for IVIG/48 sessions for SCIG.

- Training session: 4 to 6 sessions in the whole treatment period

- Follow- up: 2 sessions per year

- Intangible costs, such as costs incurred by the patient and the family stress, discomfort, and pain inflicted on the patient or the costs incurred, were not calculated.

- Cost perspective: Health service provider

- Discount rate: Given that the time considered was 1 year, in this study, discount rate was not taken into account.

\begin{tabular}{|c|c|c|}
\hline General characteristics $(\mathrm{n})$ & IVIG & SCIG \\
\hline Population $^{I}$ & 738 & 652 \\
\hline $\operatorname{SIgL}^{2}$ (efficacy), SE $^{3}$ (safety) & & \\
\hline Mean $\operatorname{SIgL}^{4}(g / l)$ & 8.54 & 9.59 \\
\hline Median SIgL (g/l) & 8.18 & 8.77 \\
\hline Costs (per year) $(\$)$ & & \\
\hline Mean hospital & 943 & - \\
\hline Mean personnel & 576 & 62 \\
\hline Mean materials and medical supplies & 73 & 68 \\
\hline Total mean direct costs & 1592 & 131 \\
\hline
\end{tabular}



Fig. 1. Decision tree for comparing IVIG and SCIG in PID patients 


\begin{tabular}{|c|c|c|c|c|}
\hline Items (Unit cost \$) & & $\operatorname{IVIG}^{I}(\$)$ & \multicolumn{2}{|c|}{$\mathrm{SCIG}^{2}(\$)$} \\
\hline Immunoglobulin & & - & \multicolumn{2}{|c|}{-} \\
\hline Personnel & & 499.35 & \multicolumn{2}{|c|}{62.21} \\
\hline Materials and medical supplies & & 62.17 & \multicolumn{2}{|c|}{58.6} \\
\hline Cost of a unit treatment services & & 152.50 & \multicolumn{2}{|c|}{63.43} \\
\hline \multicolumn{5}{|c|}{ Table 3. Parameters for sensitivity analysis } \\
\hline \multirow[t]{2}{*}{ Items } & \multicolumn{2}{|c|}{ Base case parameters $(\$)$} & \multicolumn{2}{|c|}{ Sensitivity analysis range $(\$)$} \\
\hline & IVIG & SCIG & IVIG & SCIG \\
\hline Immunoglobulin & - & - & - & - \\
\hline \multicolumn{5}{|c|}{${ }^{I}$ Session of treatment, ranging from 12 to 16 sessions for IVIG/48 to 64 sessions for SCIG } \\
\hline \multicolumn{5}{|c|}{ Table 4. Parameters for sensitivity analysis (yearly costs) } \\
\hline Items & Scenario $\mathrm{A}^{I}$ & ${\text { Scenario } \mathrm{B}^{2}}^{2}$ & Scenario $\mathrm{C}^{3}$ & Scenario $\mathrm{D}^{4}$ \\
\hline \multirow{2}{*}{$\begin{array}{cc}\text { Mean } \operatorname{Cost}^{5} & \text { IVIG } \\
& \text { SCIG } \\
\end{array}$} & 1592 & 1534 & 1497 & 1584 \\
\hline & 131 & 124 & 131 & 124 \\
\hline $\begin{array}{l}{ }^{I} \text { Scenario A: Fixed hospital, personn } \\
{ }^{2} \text { Scenario B: } 10 \% \text { reduction in person } \\
{ }^{3} \text { Scenario C: } 10 \% \text { reduction in hospit } \\
{ }^{4} \text { Scenario D: } 10 \% \text { reduction in the co } \\
{ }^{5} \text { Mean Cost: } 12 \text { to } 16 \text { sessions of IVI }\end{array}$ & $\begin{array}{l}\text { dical supply co } \\
\text { cal supplies } \\
48 \text { to } 64 \text { sessio }\end{array}$ & & & \\
\hline
\end{tabular}

\section{Cost-effectiveness analysis}

As shown in the cost calculation in Table 2, the unit cost of IVIG treatment (with an average time of 4-6 hours per session) was estimated to be $\$ 152.5$. Given the duration of treatment required to achieve effectiveness indicators (12 sessions with monthly intervals), the total cost for the first year of treatment was $\$ 1370$. The unit cost of the alternative intervention (SCIG) was estimated to be $\$ 63.43$. Given the duration of treatment required to achieve effectiveness indicators (48 sessions with weekly intervals), total costs for the first year of treatment was $\$ 121$. Moreover, cost of each therapy method was different based on the frequency of infusion sessions and duration of infusion.

\section{Sensitivity analysis}

The results of sensitivity analysis in Tables 3 and 4 indicated that changes in the number of infusion sessions affected the yearly cost of treatment, while changes in cost items (fixed hospital, personnel, and medical supply) had little effect on the final treatment cost.

Reduction in the cost of hospital had the greatest effect on the total costs than other cost items. Their relative importance is presented on a tornado diagram (Fig. 2).

\section{Incremental cost-effectiveness ratio (ICER)}

The results of meta-analysis were used to calculate ICER for adverse effects and immunoglobulin (Ig) levels. The standard mean difference was 0.366 and the odds ratio (OR) was 0.497 . The ICER for adverse ef-

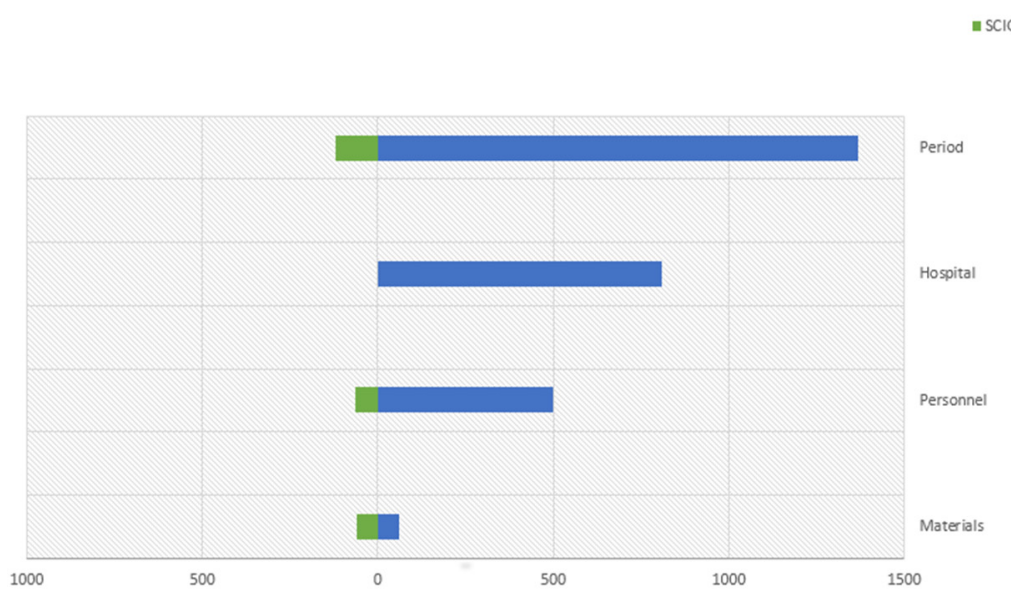

Fig. 2. Tornado diagram showing the relative of material, personnel, hospital and infusion period on total costs (Total cost difference SCIGIVIG(\$ per year). 


\begin{tabular}{|c|c|c|c|c|c|}
\hline Items & & 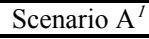 & Scenario $\mathrm{B}^{2}$ & Scenario $\mathrm{C}^{3}$ & Scenario $\mathrm{D}^{4}$ \\
\hline Mean Cost ${ }^{5}$ & IVIG & 1592 & 1534 & 1497 & 1584 \\
\hline \multirow{3}{*}{ ICER } & SCIG & 131 & 124 & 131 & 124 \\
\hline & SIgL (SMD) $)^{6}$ & 4348 & 4195 & 4067 & 4347 \\
\hline & $\mathrm{SE}(\mathrm{OR})^{7}$ & 2939 & 2836 & 2750 & 2939 \\
\hline
\end{tabular}

${ }^{1}$ Scenario A: Fixed hospital, personnel, and medical supply costs

${ }^{2}$ Scenario B: $10 \%$ reduction in personnel costs

${ }^{3}$ Scenario C: $10 \%$ reduction in hospital costs

${ }^{4}$ Scenario D: $10 \%$ reduction in the cost of medical supplies

${ }^{5}$ Mean Cost: 12 to 16 sessions of IVIG therapy/48 to 64 sessions of SCIG therapy

${ }^{6}$ Standard mean difference (for efficacy index): 0.336

${ }^{7}$ Odd ratio (for safety index): 0.497

fects and serum Ig level can be calculated as follows. The results for mean cost are presented in Table 5.

The ICER indicates that on average, $1 \%$ increase in Ig level reduces costs by $\$ 4348$. In other words, using the new intervention (SCIG) can increase serum Ig level, while reducing costs by $\$ 4348$. Moreover, $1 \%$ reduction in adverse effects reduces costs by $\$ 2939$, meaning that using the new intervention can reduce systemic adverse effects, while reducing costs by $\$ 2939$.

\section{Comparing ICER for serum Ig level and adverse effects with GDP per capita}

Iran's GDP was $\$ 4,916.10$ in the year of the study (23). Since the ICERs calculated for effectiveness indicators (\$2939 for adverse effects and \$4348 for Ig level) were lower than Iran's GDP per capita, it could be concluded that the new intervention is cost-effective. The results of sensitivity analysis also support these findings. Due to the limited number of studies in this area, no definitive conclusion can be drawn about the higher effectiveness of SCIG, and there is a need for more randomized clinical trials. However, greater ease and satisfaction in patients who used this type of treatment can indicate its advantage over IVIG for PID patients.

\section{Discussion}

The present research was the first economic assessment of IVIG versus SCIG in Iranian PID patients. The results of the meta-analysis suggested the effectiveness of SCIG in increasing serum Ig levels and reducing infection rate. Although it was associated with more adverse effects (which are mild or moderate), SCIG reduced systemic adverse effects. Overall, given the results of studies conducted on the safety and effectiveness of SCIG and the results of the meta-analysis, it can be argued that SCIG can be more effective than IVIG administration and can lead to higher satisfaction among the patients. The results of cost-effectiveness analysis revealed that the total cost of IVIG and SCIG per year was $\$ 1370$ and $\$ 121$, respectively. The cost items were the costs of hospital, personnel, and medical supplies; however, the cost of Ig dose was not taken into account due to similar doses used in both methods. In certain subgroups of patients, SCIG can be a replacement for IVIG with equal effectiveness and less systemic adverse effects (24-29). The reason is probably the more frequent infusions with less volume and slow absorption into intra- and extravascular compart- ment (30). In fact, infusion of lesser amount of Ig in shorter intervals reduces the frequency of systemic reactions $(21,31,32)$ and can improve the quality of life of the patients (33-35). Moreover, home-based SCIG reduces the annual health care costs of the patients (11, $16,36)$ and is safer and easier compared to other Ig replacement methods $(28,37,38)$.

The results of our study are consistent with those of previous studies in Canada, Germany, and Sweden with decreasing $74 \%, 82 \%$, and $55 \%$ of costs, respectively, done considering their health care systems' perspectives $(11,12,37)$. An American study also revealed $88 \%$ cost reduction in SCIG administration at home compared with IVIG delivery at hospital. However, the study revealed no cost differences between the 2 methods if both were administered at home (13).

Our results also revealed that administrating SCIG decreases costs in the first year treatment than IVIG delivery with the same dose of immunoglobulin. Previous studies indicated similar results, including a study in Canada, which found that because of needed equipment in subcutaneous administration compared with hospital costs of intravenous delivery, there was a slight cost difference between the 2 methods. Moreover, a study in France indicated that immunoglobulin delivery at home is less costly in both subcutaneous and intravenous methods (14). Furthermore, another study in France found that SCIG delivery decrease costs slightly (25 percent) than IVIG administration (15). A Japanese study also showed that moving from IVIG to SCIG has pharm economics benefits by decreasing hospital visits (16).

\section{Limitations}

Our study had some limitations. First, because of the absence of SCIG method in Iran, we conducted economic modeling to estimate SCIG method costs; the cost items were extracted from previous studies, and we calculated each item cost in Iran based on them. Moreover, to estimate costs of SCIG method, we just considered rapid push administration, while reflecting costs of pump infusion can have impressive effects on the results of the study. Finally, as mentioned in previous sections, we conducted this study from health care providers' perspective, and thus from this point of view, some costs, such as patients' travelling and indirect costs, should be omitted. However, this can have slight effects on the total cost of SCIG administration considering the flexibility of home- based treatment. 


\section{Conclusion}

The results of this study revealed that home-based SCIG is less expensive than hospital-based IVIG. The calculated ICER suggests that SCIG is more costeffective than IVIG, as it not only reduces costs with higher effectiveness compared with IVIG, but also reduces systemic adverse effects. In addition, SCIG is necessary for patients with chronic kidney disease or those without intravenous access. Currently, in most cases, SCIG is used as a complementary therapy for IVIG, as patients initially need intravenous infusion, and after some time, they can switch to SCIG. Therefore, it is recommended to use SCIG in special cases to reduce systemic adverse effects and infections and increase the quality of life of the patients.

\section{Acknowledgements}

This work was supported by Iran University of Medical Sciences (IUMS/SHMIS_2015/7).

\section{Conflict of Interests}

The authors declare that they have no competing interests.

\section{References}

1. Borte S, Wang N, Óskarsdóttir S, von Döbeln U, Hammarström L. Newborn screening for primary immunodeficiencies: beyond SCID and XLA. Ann N Y Acad Sci. 2011;1246(1):118-30.

2. Errante PR, Condino-Neto A. primary immunodeficiency diseases in Latin America: epidemiology and perspectives: INTECH Open Access Publisher; 2012.

3. Puck JM. Neonatal screening for severe combined immunodeficiency. Curr Opin Pediatr. 2011;23(6):667-73.

4. Al-Herz W, Bousfiha A, Casanova JL, Chapel H, Conley ME, Cunningham-Rundles C, et al. Primary immunodeficiency diseases: an update on the classification from the international union of immunological societies expert committee for primary immunodeficiency. Front Immunol. 2011;2.

5. Rezaei N, Abolhassani H, Aghamohammadi A, Ochs HD. Indications and safety of intravenous and subcutaneous immunoglobulin therapy. Expert Rev Clin Immunol. 2011;7(3):301-6.

6. Peter JG, Chapel H. Immunoglobulin replacement therapy for primary immunodeficiencies. Immunotherapy. 2014;6(7):853-69.

7. Wasserman RL. A new intravenous immunoglobulin (BIVIGAM ${ }^{\circledR}$ ) for primary humoral immunodeficiency. Expert Rev Clin Immunol. 2014;10(3):325-37.

8. Berger M. Adverse effects of IgG therapy. J Allergy Clin Immunol Pract. 2013;1(6):558-66.

9. Chapel HM, Spickett GP, Ericson D, Engl W, Eibl MM, Bjorkander J. The comparison of the efficacy and safety of intravenous versus subcutaneous immunoglobulin replacement therapy. J Clin Immunol. 2000;20(2):94-100.

10. Membe SK, Ho C, Cimon K, Morrison A, Kanani A, Roifman CM. Economic assessment of different modalities of immunoglobulin replacement therapy. Immunol Allergy Clin North Am. 2008; 28(4):861-74.

11. Martin A, Lavoie L, Goetghebeur M, Schellenberg R. Economic benefit of subcutaneous rapid push versus intravenous immunoglobulin infusion therapy in adult patients with primary immune deficiency. Allergy Asthma Clin Immunol. 2012;8(Suppl 1): A20.

12. Högy B, Keinecke HO, Borte M. Pharmacoeconomic evaluation of immunoglobulin treatment in patients with antibody deficiencies from the perspective of the German statutory health insurance. Eur J Health Econ. 2005;6(1):24-9.

13. Liu Z, Albon E, Hyde C, Collaboration WMHTA. The effectiveness and cost effectiveness of immunoglobulin replacement therapy for primary immunodeficiency and chronic lymphocytic leukaemia: a systematic review and economic evaluation: University of Birmingham, Department of Public Health and Epidemiology; 2005.

14. Haddad L, Perrinet M, Parent D, Leroy-Cotteau A, Toguyeni E, Condette-Wojtasik G, et al. [Economic evaluation of at home subcutaneous and intravenous immunoglobulin substitution]. Rev Med Interne. 2006;27(12):924-6.

15. Beauté J, Levy P, Millet V, Debré M, Dudoit Y, Le Mignot L, et al. Economic evaluation of immunoglobulin replacement in patients with primary antibody deficiencies. Clin Exp Immunol. 2010;160(2):240-5.

16. Igarashi A, Kanegane H, Kobayashi M, Miyawaki T, Tsutani K. Cost-minimization Analysis of IgPro20, a Subcutaneous Immunoglobulin, in Japanese Patients with Primary Immunodeficiency. Clin Ther. 2014;36(11):1616-24.

17. Pac M, Bernatowska E. Rapid push: New opportunities in subcutaneous immunoglobulin replacement therapy. Cent Eur J Immunol. 2013;38(3):388-92.

18. Lemieux R, Bazin R, Néron S. Therapeutic intravenous immunoglobulins. Mol Immunol. 2005;42(7):839-48.

19. Soler-Palacín P, Gasó-Gago I, Fernández-Polo A, Martín-Nalda A, Oliveras M, Martinez-Cutillas J, et al. Intravenous and Subcutaneous Immunoglobulin Replacement: A Two-Way Road. Optimizing Healthcare Quality in Patients with Primary Immunodeficiencies. J Clin Immunol. 2014;34(8):1015-7.

20. Shabaninejad H, Asgharzadeh A, Rezaei N, Rezapoor A. A Comparative Study of Intravenous Immunoglobulin and Subcutaneous Immunoglobulin in Adult Patients with Primary Immunodeficiency Diseases: A Systematic Review and Meta-Analysis. Expert Rev Clin Immunol. 2016:1-8.

21. Vultaggio A, Azzari C, Milito C, Finocchi A, Toppino C, Spadaro G, et al. Subcutaneous Immunoglobulin Replacement Therapy in Patients with Primary Immunodeficiency in Routine Clinical Practice: The VISPO Prospective Multicenter Study. Clin Drug Investig. 2015.

22. Martin A, Lavoie L, Goetghebeur M, Schellenberg R. Economic benefits of subcutaneous rapid push versus intravenous immunoglobulin infusion therapy in adult patients with primary immune deficiency. ransfus Med. 2013;23(1):55-60.

23. CBotIRo I. Evaluate the economic situation - mainly foreign countries Studies Department and international organizations. 2015 May 24;9.

24. Ho CT. Subcutaneous versus intravenous immunoglobulin for primary immunodeficiencies: systematic review and economic evaluation: CADTH Technol Overv; 2008.

25. Abolhassani H, Sadaghiani MS, Aghamohammadi A, Ochs HD, Rezaei N. Home-based subcutaneous immunoglobulin versus hospitalbased intravenous immunoglobulin in treatment of primary antibody deficiencies: systematic review and meta analysis. J Clin Immunol. 2012;32(6):1180-92.

26. Empson MB, Tang MLK, Pearce LKC, Rozen L, Gold MS, Katelaris $\mathrm{CH}$, et al. Efficacy, safety and pharmacokinetics of a novel

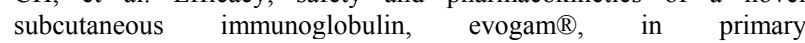
immunodeficiency. J Clin Immunol. 2012;32(5):897-906.

27. Bezrodnik L, Gómez Raccio A, Belardinelli G, Regairaz L, Díaz Ballve D, Seminario G, et al. Comparative study of subcutaneous versus intravenous IgG replacement therapy in pediatric patients with primary immunodeficiency diseases: A multicenter study in Argentina. J Clin Immunol. 2013;33(7):1216-22.

28. Shapiro R. Subcutaneous immunoglobulin (16 or 20\%) therapy in obese patients with primary immunodeficiency: A retrospective analysis of administration by infusion pump or subcutaneous rapid push. Clin Exp Immunol. 2013;173(2):365-71.

29. Saeedian M, Randhawa I. Immunoglobulin Replacement Therapy: A Twenty-Year Review and Current Update. Int Arch Allergy Immunol. 2014;164(2):151-66.

30. Bonilla FA. Intravenous immunoglobulin: adverse reactions and management. J Allergy Clin Immunol. 2008;122(6):1238-9.

31. Wasserman RL, Melamed I, Stein MR, Gupta S, Puck J, Engl W, et al. Recombinant human hyaluronidase-facilitated subcutaneous infusion of human immunoglobulins for primary immunodeficiency. $\mathrm{J}$ Allergy Clin Immunol. 2012;130(4):951-7. e11.

32. Kanegane H, Imai K, Yamada M, Takada H, Ariga T, Bexon M, et al. Efficacy and safety of IgPro20, a subcutaneous immunoglobulin, in Japanese patients with primary immunodeficiency diseases. J Clin Immunol. 2014;34(2):204-11.

33. Nicolay U, Kiessling P, Berger M, Gupta S, Yel L, Roifman CM, et al. Health-related quality of life and treatment satisfaction in North 
American patients with primary immunedeficiency diseases receiving subcutaneous IgG self-infusions at home. $\mathrm{J}$ Clin Immunol. 2006;26(1):65-72.

34. Aghamohammadi A, Montazeri A, Abolhassani H, Saroukhani S, Pourjabbar S, Tavassoli $\mathrm{M}$, et al. Health-related quality of life in primary antibody deficiency. Iran J Allergy Asthma Immunol. 2011;10(1):47-51.

35. Wasserman RL. Overview of recombinant human hyaluronidasefacilitated subcutaneous infusion of $\mathrm{IgG}$ in primary immunodeficiencies. Immunotherapy. 2014;6(5):553-67.

36. Fasth A, Nyström J. Quality of life and health-care resource utilization among children with primary immunodeficiency receiving home treatment with subcutaneous human immunoglobulin. J Clin Immunol. 2008;28(4):370-8.

37. Subcutaneous immunoglobulin replacement in patients with primary antibody deficiencies: safety and costs. Lancet February. 1995;345(8946):365-9.

38. Efficacy, Safety, and Pharmacokinetics of a $10 \%$ Liquid Immune Globulin Preparation (Gammagard Liquid, 10\%) Administered Subcutaneously in Subjects with Primary Immunodeficiency Disease. J Clin Immunol June. 2011;31(3):323-31. 